\title{
Evaluation of smartphone-integrated magnetometers in detection of safe electromagnetic devices for use near programmable shunt valves: a proof-of-concept study
}

\author{
*Smruti K. Patel, MD, ${ }^{2,3}$ Jorge Zamorano-Fernández, MD, ${ }^{1}$ Carlie McCoy, ${ }^{3}$ and Jesse Skoch, MD ${ }^{2,3}$ \\ ${ }^{1}$ Department of Neurosurgery, Hospital Infantil Universitario Niño Jesús, Madrid, Spain; ${ }^{2}$ Department of Neurosurgery, University \\ of Cincinnati College of Medicine; and 'Division of Pediatric Neurosurgery, Cincinnati Children's Hospital Medical Center, \\ Cincinnati, Ohio
}

OBJECTIVE External magnetic forces can have an impact on programmable valve mechanisms and potentially alter the opening pressure. As wearable technology has begun to permeate mainstream living, there is a clear need to provide information regarding safety of these devices for use near a programmable valve (PV). The aim of this study was to evaluate the magnetic fields of reference devices using smartphone-integrated magnetometers and compare the results with published shunt tolerances.

METHODS Five smartphones from different manufacturers were used to evaluate the magnetic properties of various commonly used $(n=6)$ and newer-generation $(n=10)$ devices using measurements generated from the internal smartphone magnetometers. PV tolerance testing using calibrated magnets of varying field strengths was also performed by smartphone magnetometers.

RESULTS All tested smartphone-integrated magnetometers had a factory sensor saturation point at around $5000 \mu T$ or 50 Gauss (G). This is well below the threshold at which a magnet can potentially deprogram a shunt, based on manufacturer reports as well as the authors' experimental data with a threshold of more than $300 \mathrm{G}$. While many of the devices did saturate the smartphone sensors at the source, the magnetic flux density of the objects decreases significantly at 2 inches.

CONCLUSIONS The existence of an upper limit on the magnetometers of all the smartphones used, although well below the published deprogramming threshold for modern programmable valves, does not allow us to give precise recommendations on those devices that saturate the sensor. Based on the authors' experimental data using smartphoneintegrated magnetometers, they concluded that devices that measure $<40 \mathrm{G}$ can be used safely close to a PV.

https://thejns.org/doi/abs/10.3171/2020.10.PEDS20727

KEYWORDS programmable valve; hydrocephalus; shunt; cerebrospinal fluid; magnetometer; smartphone; surgical technique

$\mathrm{H}$ YDROCEPHALUS is one of the most common neurosurgical disorders, and, with an estimated incidence by region of 68-316 per 100,000 births, it is the most commonly encountered pathology for the pediatric neurosurgeon. ${ }^{1}$ Hydrocephalus remains a chronic disease, and, among the different treatment options, lifetime shunting remains the most frequently used technique. Differential-pressure valves are the most commonly used CSF modulators in contemporary practice. These valves passively open to CSF flow when the differential pressure between the ventricular and distal compartment exceeds a preestablished fixed opening pressure. The biomechanics of CSF flow can vary over the course of the disease, and the valve opening pressure deemed appropriate at the moment of surgery can change over time or be proven incorrect on postoperative evaluation. To address this, programmable differential-pressure valves were developed in the 1980s and further popularized in the 1990s. ${ }^{2}$ Programmable valves (PVs) incorporate a mechanism regulated by a spring that can be adjusted noninvasively to a range of operating pressures by using a magnetic field (MF) transmitted through the skin. There are currently several PV models from different manufacturers.

Unnoticed external forces can have an influence over PV mechanisms and modify the opening pressure setting. ${ }^{3}$ MFs produced by many devices can often be a concern for 
patients and parents of children with shunt-treated hydrocephalus who have PVs, since strong MFs from external magnetic sources and implanted medical devices that use magnets can cause an unintended change in their valve setting. Although devices from the most recently developed generation have been designed with greatly improved resistance to MFs, ${ }^{4-8}$ devices sensitive to field strengths of more than 90 Gauss $(\mathrm{G})$ are still commonly implanted and will exist in patients for decades to come. A wide variety of medical ${ }^{9,10}$ and consumer ${ }^{8,11-17}$ devices with MFs have been reported to interfere with the PV mechanism and setting in vitro and in vivo (Fig. 1).

However, it is known that magnetic flux density decreases in inverse proportion to the square of the distance from the magnetic pole, and it is possible that the use of many of these devices will be safe in real-life scenarios, where valves are placed below the scalp surface and a few centimeters away from the ear. ${ }^{18-20}$ As wearable technology continues to permeate mainstream living, blanket restrictions on the use of devices capable of generating small MFs near shunt hardware will become an increasing burden on patients with these devices. There is a clear need to provide specific answers to patient questions about technology restrictions. Nevertheless, due to the vast number of MF-generating devices on the market, the exponential release rate of new products, and the lack of accessible information regarding their MF strengths, specific timely recommendations are hardly ever available. Given these insufficient data, patients are generally advised to simply restrict their use in the vicinity of a PV. Universal restriction of the use of such devices may have a negative impact on patients' quality of life.

The aim of this study was to evaluate the MFs of reference devices using smartphone-integrated magnetometers and compare the results with those of published shunt tolerances to determine the potential to translate existing precautionary data to applicable values from smartphone magnetometers. Furthermore, we sought to assess the consistency of measurements across different varieties of smartphones and perform our own programmable valve tolerance testing using calibrated magnets of varying field strengths as measured by smartphone magnetometers.

\section{Methods}

\section{Using a Smartphone Magnetometer}

We used 5 different types of smartphones from different manufacturers to evaluate the magnetic properties of various objects by using measurements generated from the internal magnetometers of each of these devices. Magnetometers can be used to detect and quantify magnetic flux densities. Nearly all modern smartphones contain Hall effect-chip-integrated magnetometers that are principally used as compasses and can determine the handset's orientation in 3 dimensions with field measurements in the $\mathrm{x}-, \mathrm{y}-$, and $\mathrm{z}$-axes. ${ }^{21}$ Smartphone sensors, including magnetometers, can be utilized with open-source free apps like the phyphox ${ }^{22}$ app used in the current study. The phyphox app is available for the most commonly used mobile phone operating systems and can be used to detect the electromagnetic field surrounding a

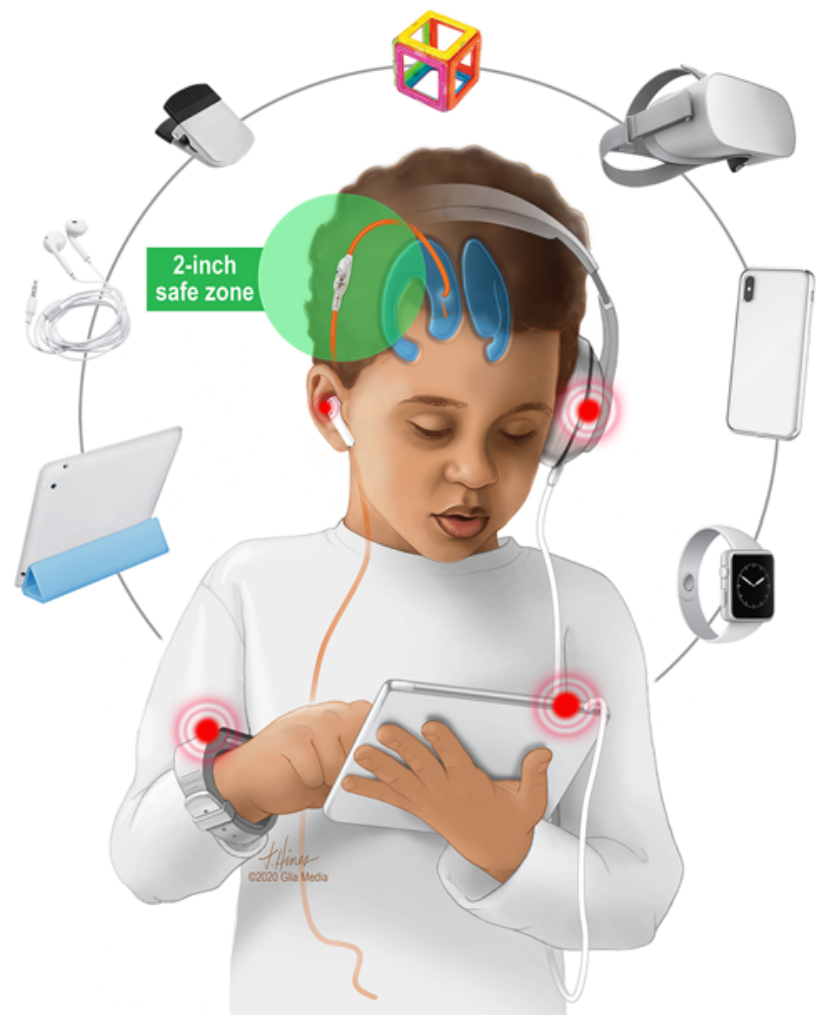

FIG. 1. A wide variety of consumer devices have electromagnetic properties that can interfere with programmable valve mechanisms. Current recommendations suggest keeping these products 2 or more inches away from the location of the programmable valve as shown in this original illustration. Published with permission from Glia Media. Artist: Tonya Hines, CMI. Figure is available in color online only.

particular object. ${ }^{23}$ Within the app, the user is allowed to select the "Magnetometer" function, which generates the raw data from the magnetometer within the device. For simplicity purposes, we recommend using the "Absolute" or "Multi" functions. It is critical that, prior to completing any of the next steps (including location, calibration, and obtaining measurements), any casings on the devices must be removed to achieve accurate readings.

\section{Locating the Magnetometer on Smartphones}

The main manufacturer of Hall effect-chip-integrated magnetometers is Asahi Kasei Microdevices (AKM Corp.); although different smartphone-integrated magnetometer models exist, they are all very similar in construction and functioning. During our research, we found tested smartphones to have these sensors located variably across makes and models (Table 1). Accurately locating the magnetometer is straightforward and can be achieved with any thin, weakly magnetized object (such as an unfurled paperclip dragged repeatedly over a magnet in the same direction). Given the rapid decay of MF strength, it is important to accurately locate the sensor prior to obtaining measurements. Utilizing this technique has resulted in comparable measurements among different makes and models examined in this study. 
TABLE 1. Approximate sensor location, type of magnetometer, and maximum range of magnetometer for each smartphone used in this study

\begin{tabular}{lccc}
\hline \multicolumn{1}{c}{ Device } & Approximate Sensor Location & Magnetometer & Maximum Range (G) \\
\hline iPhone X & Bottom rt corner of device & $-{ }^{*}$ & $45-60$ \\
\hline Sony Xperia & Bottom rt corner of device & AKM ak09915 & 49.11 \\
\hline Samsung Galaxy S9 & Top It corner of device & AKM ak09916C & 49.12 \\
\hline Xiaomi Mi A9 & Top It corner of device & AKM ak09918 & 49.11 \\
\hline Xiaomi Redmi Note 8 & Top It corner of device & AKM ak09918 & 49.11 \\
\hline
\end{tabular}

* Manufacturer information regarding the type of magnetometer used for the iPhone $X$ is unavailable.

\section{Calibration of Smartphone Magnetometer}

The Earth's inherent MF is on the order of $50 \mu \mathrm{T}$ or 0.5 G. ${ }^{3}$ The values generated by magnetometers are in units of micro-Teslas $(\mu \mathrm{T})$. The ambient or baseline measurement on magnetometers across tested smartphone devices in the study ranged between 20 and $100 \mu \mathrm{T}$. For the purposes of the study and to easily compare across devices and manufacturer values, we have converted all measurements from the magnetometer to Gauss values. To ensure accuracy, we recommend that, prior to using the magnetometer to measure the MF of an object, each device is calibrated. The process of compass calibration varies for each type of smartphone. For example, on the iOS devices, the compass is calibrated by the following selections: Click on Settings icon $>$ Select Privacy $>$ Select Location Services $>$ Select System Service > Turn Compass Calibration ON. If the ambient measurement on the smartphone still reads higher than typical baseline values $(>100 \mu \mathrm{T})$, then the phone should be restarted and the app reopened prior to performing any more measurements.

\section{Obtaining MF Measurements}

Once the location and calibration of the smartphone magnetometer is confirmed, the phyphox app on each of the devices can be used to generate source and 2-inch measurements for each of the objects of interest. After the application is opened, the raw sensor labeled "Magnetometer" is selected. Then, either the "Absolute" or "Multifunction" is selected. Within these functions, there is a "play" and "pause" function. When the user is ready to obtain source measurements, the "play" function is selected, and the object of interest is held directly at the location of the magnetometer in the device. The absolute, consistent, maximal value generated by the magnetometer is then recorded for that object. For the majority of the devices, the center of the magnet or the location of the speaker (which contains a magnet) was the ideal area to test for maximal exposure. For 2-inch measurements, we used a template (Fig. 2) in which the smartphone magnetometer is placed at the center of the template. The object of interest is then placed 2 inches away (designated by a bold black line), the "play" function is selected in the app on the device, and the object is rotated around the magnetometer to obtain the absolute, consistent, maximal value of the MF.

\section{Programmable Valve Tolerance Testing}

For initial threshold testing, a strong magnet $(1500 \mathrm{G})$ was used to determine what type of movement would alter the valve settings on both a Codman Certas Plus (Integra LifeSciences) and a Strata II (Medtronic) valve. Several movements were performed with the magnet just above each of the valves in the following manner: vertical swipes, horizontal swipes, diagonal swipes, and circular motions. Each type of movement was tested on the valve in 3 trials of 50 swipes and 3 trials of 100 swipes. Before and after each trial, the valve setting was measured and recorded, and the two values were compared to determine if the valve setting changed. The circular motions proved to be the most reliable movement to alter the valve settings, producing a change $80 \%$ of the time. It is postulated that the likelihood of valve alteration during these trials was actually $100 \%$, but $20 \%$ of the time, it changed back to the original setting (there are 5 possible setting positions on this valve). Based on these data, circular motions were used to test calibrated magnets on the valve. This is consistent with a prior report that showed that a rotational component of the magnet in devices can cause reprogramming of a valve at the source (i.e., direct contact at $0 \mathrm{~mm}){ }^{19}$

During this preliminary testing, we found that compared with the Codman Certas Plus valve, the Strata II valve was more likely to have valve setting changes. The setting on the Codman Certas Plus valve was not altered in any of the preliminary trials with the strong magnet. Therefore, we objectively moved forward with further calibrated magnet testing with a ring and a disc/cylinder magnet using the Strata II valve. Using a field strength calculator provided by the magnet manufacturer, which we experimentally verified (http://www.kjmagnetics.com/ fieldcalculator.asp), we tested a range of field strengths by attaching the magnets to wooden dowels with modeling clay. The tip of the dowel could then contact the top of the valve packaging to maintain a consistent minimum distance during magnet movement.

\section{Results}

\section{Smartphone Magnetometer Readings}

For this study, we used 5 different smartphones to measure MF strengths. These included the iPhone X (Apple Inc.), Sony Xperia (Sony Mobile Communications Inc.), Samsung Galaxy S9 (Samsung Electronics Co. Ltd.), Xiaomi Mi A9 (Xiaomi Corp.), and Xiaomi Redmi Note 8 (Xiaomi Corp.). The location of the inte- 


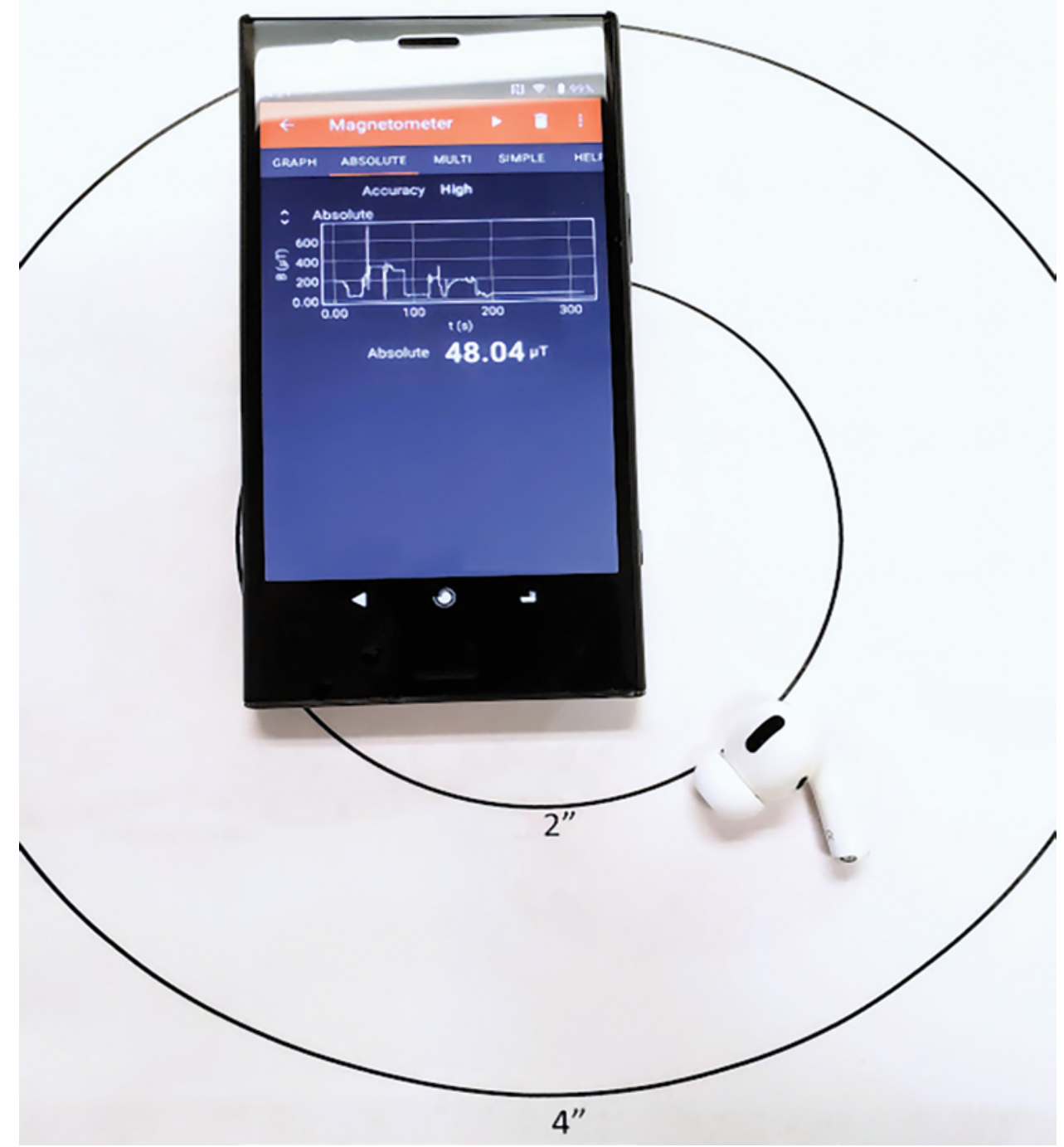

FIG. 2. Template used for obtaining 2-inch measurements with a smartphone-integrated magnetometer. The magnetometer is placed in the center of the template and the object of interest and rotated in a circular fashion at 2-inch radius markings. Figure is available in color online only.

grated magnetometers was confirmed, and the compass calibration was completed. Baseline ambient measurements were obtained on all devices to ensure accuracy. In addition, the maximal range of the magnetometer was also noted (Table 1). We tested the MF strengths of commonly used objects at the source, including a refrigerator magnet, magnetic building toy, kitchen timer, airline-issued headphones, an iPad Smart Cover (Apple Inc.), and an iPad tablet (Apple Inc.). The results are shown in Table 2 ; overall, the data suggest that most smartphone magnetometers reach a threshold between 40 and $60 \mathrm{G}$. All of these measurements were then repeated at 2 inches away from the source using each of the smartphone devices, and the results showed that the magnetic flux density of the objects decreases significantly at 2 inches (Table 2). Mean MF strength readings from these commonly used objects were then compared with the previously published manufacturer thresholds ${ }^{24,25}$ for the Strata II and Codman Certas Plus valves (Table 3). While there was variabil- ity with regard to the manufacturers' reports for certain objects, smartphone sensors saturated appropriately for highly magnetic objects.

Seven newer-generation products were then tested (10 magnetic surfaces) using each of the smartphone-integrated magnetometers both at the source and at 2 inches (Table 4). These objects included the following: Apple wired headphones, the original AirPods and accompanying case, and AirPods Pro and accompanying case (all Apple Inc.); wireless on-ear headphones (Bose QuietComfort 35 II), Oculus Go virtual reality headset (Facebook Technologies, LLC.); the Apple Watch (Apple Inc.); and the Laugh and Learn Smart Stages Toy (Fisher-Price). The mean data showed that the majority, but not all, of these newergeneration products contain strong magnets that cannot be accurately quantified by magnetometers because of saturation of the magnetometer when measured at the source. There was a precipitous falloff at 2 inches in all of the measurements across all the smartphone devices. 
TABLE 2. MF strength measurements for commonly used objects at source and at 2 inches using 5 different smartphone magnetometers

\begin{tabular}{|c|c|c|c|c|c|c|c|}
\hline Type of Device & Specific Description & Sensor Distance & iPhone X & Sony Xperia & Samsung Galaxy S9 & Xiaomi Mi A9 & Xiaomi Redmi Note 8 \\
\hline \multirow{2}{*}{$\begin{array}{l}\text { Refrigerator } \\
\text { magnet }\end{array}$} & \multirow{2}{*}{$\begin{array}{l}\text { Oxo SoftWorks Fridge Mag- } \\
\text { net Clip, model \#108911 }\end{array}$} & Source & $40^{*}$ & $48.96^{*}$ & $49.78^{*}$ & $51.3^{*}$ & $54^{*}$ \\
\hline & & 2 inches & 1.4 & 1.32 & 2.62 & 2.4 & 1.7 \\
\hline \multirow{2}{*}{ Magnetic toy } & \multirow{2}{*}{ Magformers, model \#63076 } & Source & $40^{*}$ & $48.9^{*}$ & $45.35^{*}$ & $51^{*}$ & $53^{*}$ \\
\hline & & 2 inches & 1.2 & 1.26 & 1.6 & 2.8 & 1.6 \\
\hline \multirow{2}{*}{ Kitchen timer } & \multirow{2}{*}{ CDN, model \#TM12 } & Source & 6.25 & 4.59 & 5.97 & 6.7 & 3.8 \\
\hline & & 2 inches & 0.49 & 0.4 & 0.5 & 0.55 & 0.55 \\
\hline \multirow{2}{*}{$\begin{array}{l}\text { Airline-issued } \\
\text { earbuds }\end{array}$} & \multirow{2}{*}{ Billboard wired earbuds } & Source & 4.75 & 3.75 & 3.96 & 6.2 & 3.15 \\
\hline & & 2 inches & 0.43 & 0.12 & 0.12 & 0.54 & 0.53 \\
\hline \multirow{2}{*}{$\begin{array}{l}\text { iPad Smart } \\
\text { Cover }\end{array}$} & \multirow{2}{*}{ Apple iPad Smart Cover } & Source & $40^{*}$ & $48.9^{*}$ & $43.2^{*}$ & $51.2^{*}$ & $52^{*}$ \\
\hline & & 2 inches & 1.2 & 1.3 & 1.41 & 2.5 & 0.5 \\
\hline \multirow{2}{*}{ ¡Pad tablet } & \multirow{2}{*}{ Apple iPad Pro } & Source & $40^{*}$ & $50.22^{*}$ & $42.5^{*}$ & $53^{*}$ & $53^{*}$ \\
\hline & & 2 inches & 0.62 & 1.56 & 1.30 & 3.01 & 0.86 \\
\hline
\end{tabular}

All measurements were obtained in micro-Teslas and converted to Gauss.

* Out of reliable range for smartphone magnetometers; value is likely significantly higher than recorded.

\section{Programmable Valve Tolerance Testing}

The electromagnetic field strengths for each calibrated magnet at varying distances are shown in Table 5. As expected, the Gauss level of emission from each magnet decreased with increasing distance from the shunt valve (Fig. 3). The Strata II valve was set to 1.5 prior to all trials. Both magnets generated a significant field strength at source and changed the Strata II valve setting every time. However, the weaker ring magnet did not appear to alter the valve settings at any further distances. The disc/cylinder magnet, however, did change the Strata II valve setting at a distance of 0.6 inches or less, consistently.

\section{Discussion}

The aim of this study was to address the use of a free, accessible, open-source app to exploit smartphone-integrated magnetometers as tools to detect MFs emitted by common consumer devices and determine whether or not they pose a risk of deprogramming PVs. To our knowl- edge, this is the first study of its kind to utilize this type of accessible technology. The risk of deprogramming PVs with electromagnetic devices and toys is a common concern for patients, parents, and providers alike. While the US FDA recently released general recommendations related to this topic and the consequences of unintended changes to CSF shunt valve settings that, if unchecked, may become serious healthcare concerns, ${ }^{26}$ we still lack specific recommendations for most of the consumer and professional devices used.

In conducting this study, we discovered that all tested smartphone-integrated magnetometers have a factory sensor saturation point at around $5000 \mu \mathrm{T}$ or $50 \mathrm{G}$. This is well below the threshold that a magnet can potentially deprogram a shunt based on manufacturer reports (threshold of $90 \mathrm{G})^{27-29}$ as well as our own experimental data with a threshold of $>300 \mathrm{G}$ ). The existence of an upper limit on the magnetometers of all the smartphones used, although well below the published deprogramming threshold for modern PVs, does not allow us to give precise recommen-

TABLE 3. Mean magnetic field strength measurements from commonly used objects (at source) compared with manufacturers' reports

\begin{tabular}{|c|c|c|c|}
\hline Device & Mean Reading Detected by Smartphone Magnetometers (G) & Medtronic Reading $(G)^{*}$ & Codman Reading $(G) \dagger$ \\
\hline Refrigerator magnet & $47.07 \ddagger$ & 305 & NA \\
\hline Magnetic toy & $50.97 \ddagger$ & NA & 1545.6 \\
\hline Kitchen timer & 5.46 & NA & 1623.7 \\
\hline Earphones & 4.46 & 350 & 221.9 \\
\hline Magnetic tablet case & $48.03 \ddagger$ & 2060 & 2080.3 \\
\hline Noise-cancelling headphones & $50.25 \ddagger$ & 115 & NA \\
\hline Refrigerator door seal & $48.64 \ddagger$ & 340 & NA \\
\hline Tablet & $50.22 \ddagger$ & NA & 1118.6 \\
\hline Smartphone & $42.63 \ddagger$ & 525 & 296.7 \\
\hline
\end{tabular}

$\mathrm{NA}=$ measurement not available for comparison.

* http://www.medtronic.me/content/dam/medtronic-com/products/neurological/shunts/ns-stratamagnetic_rev-e.pdf.

† https://www.integralife.com/file/general/1551213408.pdf.

$\ddagger$ Out of reliable range for smartphone magnetometers; value is likely significantly higher than recorded. 
TABLE 4. Mean readings of newer-generation devices at source detected by smartphone magnetometers

\begin{tabular}{|c|c|c|}
\hline Device & $\begin{array}{r}\text { Mean S } \\
\text { Magn } \\
\text { Rea }\end{array}$ & $\begin{array}{l}\text { tphone } \\
\text { neter } \\
\text { (G) }\end{array}$ \\
\hline \multirow{2}{*}{ Apple wired headphones } & Source & $41.5^{*}$ \\
\hline & 2 inches & 0.66 \\
\hline \multirow{2}{*}{ Apple AirPods, original } & Source & $54.76^{*}$ \\
\hline & 2 inches & 0.66 \\
\hline \multirow{2}{*}{ Apple AirPods case, original } & Source & $50^{*}$ \\
\hline & 2 inches & 2.50 \\
\hline \multirow{2}{*}{ Apple AirPods Pro } & Source & 36.87 \\
\hline & 2 inches & 0.20 \\
\hline \multirow{2}{*}{ Apple AirPods Pro case } & Source & $60^{*}$ \\
\hline & 2 inches & 2.05 \\
\hline \multirow{2}{*}{$\begin{array}{l}\text { Wireless on-ear headphones (Bose Quiet- } \\
\text { Comfort } 35 \text { II Bluetooth, noise-cancelling) }\end{array}$} & Source & $50.25^{*}$ \\
\hline & 2 inches & 2.45 \\
\hline \multirow{2}{*}{ Oculus Go virtual reality headset } & Source & 27.33 \\
\hline & 2 inches & 2.34 \\
\hline \multirow{2}{*}{ Apple Watch, front \& sides } & Source & 8.23 \\
\hline & 2 inches & 0.34 \\
\hline \multirow{2}{*}{ Apple Watch, back } & Source & $60^{*}$ \\
\hline & 2 inches & 1.12 \\
\hline \multirow{2}{*}{ Laugh and Learn Smart Toy (Fisher-Price) } & Source & 5.085 \\
\hline & 2 inches & 0.41 \\
\hline
\end{tabular}

All measurements were obtained in micro-Teslas and converted to Gauss.

* Out of reliable range for smartphone magnetometers; value is likely significantly higher than recorded.

dations on those devices that clip, or saturate, the sensor. Those devices that measure $<4000 \mu \mathrm{T}(<40 \mathrm{G})$, on smartphone magnetometers can be used safely close to a PV or near the head region. We are investigating safe options for more accurate measurements of stronger MFs with smartphone sensors by taking advantage of the rapid decay of field strength over distance from the sensor. However, the more cautious action would be to assume devices that are able to reach $>40 \mathrm{G}$ at source measurements should not be placed near the head if a PV is present.

While some PVs are extremely resistant to strong MF noise, many relatively sensitive valves are in use today and will remain in implanted circulation for many years to come. Given the growing number of consumer and professional MF-emitting devices, including wearable technologies and the continuous release of new products, it will be impossible for systemic research to assess and make individualized recommendations for each particular device. Even current lists lack specificity, and devices may have different magnets with slightly different models. Our data indicate that carefully examined devices that do not trigger clipping $(>4000 \mu \mathrm{T})$ in direct proximity to the specific location of a smartphone magnetometer at the measured distances could be considered safe for use on the head or ears. This is currently an observation and not an endorsed recommendation. However, rather than limit patients with an overcautious ban on all devices with electromagnetic
TABLE 5. Change in Strata II shunt setting at different distances from calibrated magnets

\begin{tabular}{ccc}
\hline $\begin{array}{c}\text { Magnet Type \& } \\
\text { Distance (inches) }\end{array}$ & $\begin{array}{c}\text { Magnetic Field } \\
\text { Strength }(G)\end{array}$ & $\begin{array}{c}\text { Valve } \\
\text { Deprogrammed? }\end{array}$ \\
\hline Ring magnet & \\
\hline 0 & 1271 & Yes \\
\hline 0.25 & 461 & No \\
\hline 0.375 & 341 & No \\
\hline 0.5 & 224 & No \\
\hline 0.75 & 100 & No \\
\hline 1 & 51 & No \\
\hline 1.25 & 29 & No \\
\hline Disc magnet & & \\
\hline 0 & 3396 & Yes \\
\hline 0.5 & 631 & Yes \\
\hline 0.6 & 417 & Yes \\
\hline 0.75 & 235 & No \\
\hline 1.0 & 113 & No \\
\hline 1.25 & 60 & No \\
\hline 1.5 & 28 & No \\
\hline
\end{tabular}

Gray shading indicates changes in shunt settings.

* Outer diameter: 0.625 inches; thickness: 0.1875 inches.

fields, we submit that self-screening with a very cautious and conservative 40-G threshold using any smartphone device could allow for safe use of select technologies without waiting for third-party reports to be generated years after a technology is available. Our results were not always consistent with manufacturer published lists, likely because of difficult-to-verify variables, including manufacturer and specific device model types. Proper empirical measurement of a device's MF is arguably the most reliable indicator of shunt compatibility. Our data also emphasize the significance of keeping the magnetic source even a small distance away (i.e., 2 inches) from a PV when possible, given the steep and exponential falloff of the MF. Further investigation of the utility of smartphone magnetometry for device safety assessment is underway.

The in vitro nature of this experiment and the relatively limited number of smartphone types tested are inherent limitations of the study. In addition, the existence of a relatively low upper clipping/saturation point on all sensors used precludes accurate quantification of all but low-powered magnets. The precipitous drop-off of MF strength (Fig. 3) can also lead to biased underreported field strengths if devices are not carefully scanned precisely at the sensor location and in three dimensions. It is important to highlight several technical nuances that we discovered in this study to achieve the most precise and accurate readings. 1) All phone casings should be removed. 2) Sensor functionality can be compromised temporarily if strong fields are encountered, and, therefore, calibration of the sensor is recommended prior to testing different objects. 3) Precisely locating the magnetometer on the smartphone device is instrumental. 4) It is important to locate the MFemitting source in the object being tested. Keeping these 

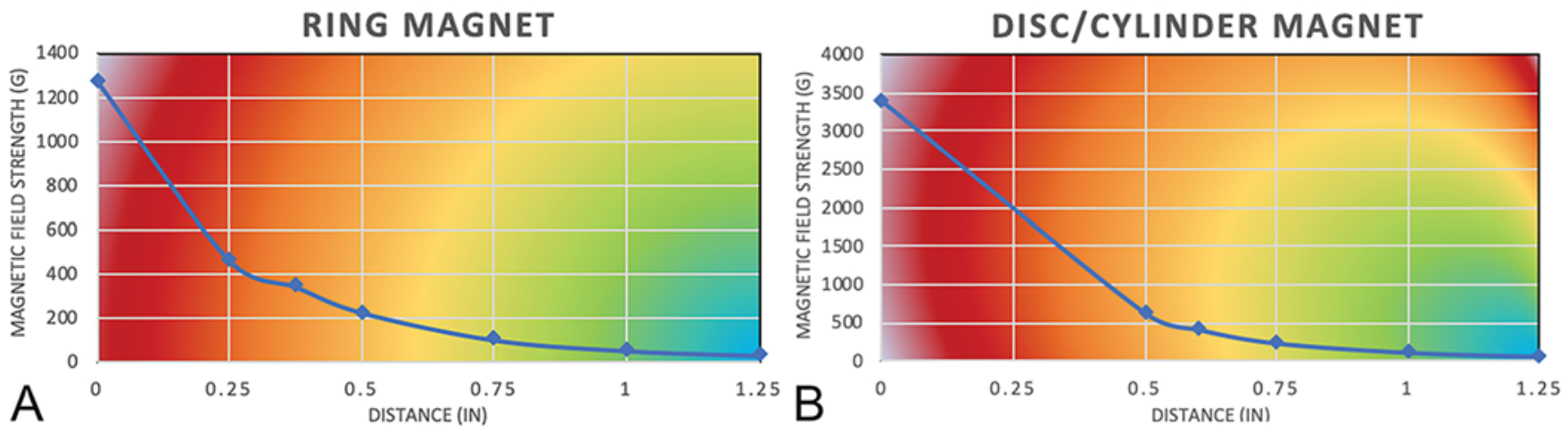

FIG. 3. Visual representation of the precipitous drop-off in MF strength seen with both the ring magnet $(\mathbf{A})$ and disc/cylinder magnet $(B)$ as the distance from the shunt valve is increased during the performance of programmable valve tolerance testing. Figure is available in color online only.

technical factors in mind, our approach appears to be sensitive and accurate in a conservative range $(<40 \mathrm{G})$ with a fairly minimal number of iterations. There is opportunity to optimize smartphone software for this application, including options to autolocate the magnetometer in a device, provide clear instructions for testing, and perhaps even allow for crowd-sourced databasing of modern devices. In addition, this pocket technology is readily and easily accessible on-hand, allowing for versatile, reliable measurements that can be obtained anywhere. It is important to note that use of these screening devices is for precautionary use only.

While there is a risk of unintended changes in PV settings due to magnet interference, the prevalence is not known. Patients should be counseled that exposure to magnets may affect valve settings in externally programmable CSF shunt systems. Patient and family education on shunt implants is paramount and usually must be administered repeatedly. At the very least, patients and families should be aware of whether or not they have a PV in place. We advocate for structured shunt education ${ }^{30}$ and have integrated patient/family usage of the patient-centric free Hydrocephalus Association HydroAssist smartphone app ${ }^{31}$ into our own practice. Our group supports the guidelines recently published by the FDA,${ }^{12}$ which recommend keeping products that contain magnets 2 or more inches away from the location of a programmable CSF shunt valve. Patients continue to be advised to use the ear opposite the shunt valve for devices that require listening, such as cellular phones and earbuds.

\section{Conclusions}

Without a patient-centric tool to assess safety, blanket restrictions on the usage of wearable technology in patients with first-generation programmable valves is the safest precaution. However, this may impact quality of life for those patients who are restricted from smartwatches, sleep monitors, virtual reality and augmented-reality headsets, headphones, and smart eyeglasses. Accessible pocket technology such as smartphone-integrated magnetometers can be used to screen safety of personal electromagnetic devices and may provide some resolution for future recommendations regarding the use of these products. A dedicat- ed instructional application and integrated crowd sourcing to database the specific devices and their MFs for proper patient usage may also be beneficial.

\section{Acknowledgments}

We thank Glia Media for their medical illustration services.

\section{References}

1. Dewan MC, Rattani A, Mekary R, et al. Global hydrocephalus epidemiology and incidence: systematic review and metaanalysis. J Neurosurg. 2019;130(4):1065-1079.

2. Aschoff A, Kremer P, Hashemi B, Kunze S. The scientific history of hydrocephalus and its treatment. Neurosurg Rev. 1999;22(2-3):67-95.

3. Strahle J, Collins K, Stetler WR Jr, et al. Effect of amusement park rides on programmable shunt valve settings. Pediatr Neurosurg. 2013;49(1):21-23.

4. Czosnyka Z, Pickard JD, Czosnyka M. Hydrodynamic properties of the Certas hydrocephalus shunt. J Neurosurg Pediatr. 2013;11(2):198-204.

5. Inoue T, Kuzu Y, Ogasawara K, Ogawa A. Effect of 3-tesla magnetic resonance imaging on various pressure programmable shunt valves. J Neurosurg. 2005;103(2)(suppl):163-165.

6. Lavinio A, Harding S, Van Der Boogaard F, et al. Magnetic field interactions in adjustable hydrocephalus shunts. $J \mathrm{Neu}$ rosurg Pediatr. 2008;2(3):222-228.

7. Moghtader D, Crawack HJ, Miethke C, et al. Assessment of MRI issues for a new cerebral spinal fluid shunt, gravitational valve (GV). Magn Reson Imaging. 2017;44:8-14.

8. Schneider T, Knauff U, Nitsch J, Firsching R. Electromagnetic field hazards involving adjustable shunt valves in hydrocephalus. J Neurosurg. 2002;96(2):331-334.

9. Jandial R, Aryan HE, Hughes SA, Levy ML. Effect of vagus nerve stimulator magnet on programmable shunt settings. Neurosurgery. 2004;55(3):627-630.

10. Lefranc M, Ko JY, Peltier J, et al. Effect of transcranial magnetic stimulation on four types of pressure-programmable valves. Acta Neurochir (Wien). 2010;152(4):689-697.

11. Anderson RC, Walker ML, Viner JM, Kestle JR. Adjustment and malfunction of a programmable valve after exposure to toy magnets. Case report. J Neurosurg. 2004;101(2)(suppl): 222-225.

12. Fujimura R, Lober R, Kamian K, Kleiner L. Maladjustment of programmable ventricular shunt valves by inadvertent exposure to a common hospital device. Surg Neurol Int. 2018;9:51.

13. He Y, Murphy RK, Roland JL, Limbrick DD Jr. Interactions 
between programmable shunt valves and the iPad 3 with Smart Cover. Childs Nerv Syst. 2013;29(4):531-533.

14. Nakashima K, Nakajo T, Kawamo M, et al. Programmable shunt valves: in vitro assessment of safety of the magnetic field generated by a portable game machine. Neurol Med Chir (Tokyo). 2011;51(9):635-638.

15. Ozturk S, Cakin H, Kurtuldu H, et al. Smartphones and programmable shunts: are these indispensable phones safe and smart? World Neurosurg. 2017;102:518-525.

16. Strahle J, Selzer BJ, Muraszko KM, et al. Programmable shunt valve affected by exposure to a tablet computer. $J \mathrm{Neu}$ rosurg Pediatr. 2012;10(2):118-120.

17. Utsuki S, Shimizu S, Oka H, et al. Alteration of the pressure setting of a Codman-Hakim programmable valve by a television. Neurol Med Chir (Tokyo). 2006;46(8):405-407.

18. Nomura S, Fujisawa H, Suzuki M. Effect of cell phone magnetic fields on adjustable cerebrospinal fluid shunt valves. Surg Neurol. 2005;63(5):467-468.

19. Pierson MJ, Wehrmann D, Albers JA, et al. Programmable shunt valve interactions with osseointegrated hearing devices. J Neurosurg Pediatr. 2017;19(4):384-390.

20. Spader HS, Ratanaprasatporn L, Morrison JF, et al. Programmable shunts and headphones: are they safe together? J Neurosurg Pediatr. 2015;16(4):402-405.

21. Arribas E, Escobar I, Suarez CP, et al. Measurement of the magnetic field of small magnets with a smartphone: a very economical laboratory practice for introductory physics courses. Eur J Phys. 2015;36(6):065002.

22. Staacks S. Talk at the Chaos Communication Congress. phyphox: physical phone experiments. December 28, 2019. Accessed December 9, 2020. https://phyphox.org/2019/12/

23. Staacks S, Hütz S, Heinke H, Stampfer C. Advanced tools for smartphone-based experiments: phyphox. Phys Educ. 2018; 53(4):045009.

24. Break Through the Uncertainty Brochure. Codman 12362173-EN Certas Plus; 2019. Accessed December 7, 2020. https:// www.integralife.com/file/general/1551213408.pdf

25. Magnetic Field Influences and Your Strata TM Valve. Medtronic EN UC201504957A; 2017. Accessed December 7, 2020. http://www.medtronic.me/content/dam/medtronic-com/ products/neurological/shunts/ns-stratamagnetic_rev-e.pdf

26. Magnetic field interference with programmable CSF shunts. FDA.gov. July 16, 2019. Accessed December 7, 2020. https:// www.fda.gov/medical-devices/cerebral-spinal-fluid-csf-shuntsystems/magnetic-field-interference-programmable-csfshunts

27. "Kronos" Valve Resistance to Spontaneous PL Setting changes. MNS Report RD9310-04. Medtronic; 1998.

28. Magnetic Inadvertent Switching. Non-homogeneous Dy- namic Magnetic Influence Characterization of the Strata NSC Valve and Codman Hakim Programmable Valve. MNS Technical Document TD-02864. Medtronic; 2004.

29. Strata II Valve Design Verification Report: Resistance to Inadvertent Switching. MNS Technical Document TD-02843. Medtronic; 2004.

30. Ackerman LL, Fulkerson DH, Jea A, Smith JL. Parent/guardian knowledge regarding implanted shunt type, setting, and symptoms of malfunction/infection. $J$ Neurosurg Pediatr. 2018;21(4):359-366.

31. HydroAssist ${ }^{\circledR}$ Mobile Application. Hydrocephalus Association. Accessed December 7, 2020. https://www.hydroassoc. org/hydroassist-mobile-application/

\section{Disclosures}

The authors would like to disclose that, for testing purposes, they graciously received a Strata II valve from Medtronic and a Codman Certas Plus valve from Integra LifeSciences and requested internal company testing data for the respective valves.

\section{Author Contributions}

Conception and design: Skoch. Acquisition of data: all authors. Analysis and interpretation of data: Skoch, Patel, ZamoranoFernández. Drafting the article: Patel, Zamorano-Fernández. Critically revising the article: Skoch, Patel, Zamorano-Fernández. Reviewed submitted version of manuscript: all authors. Approved the final version of the manuscript on behalf of all authors: Skoch Administrative/technical/material support: McCoy. Study supervision: Skoch.

\section{Supplemental Information Previous Presentations}

The preliminary results and abstract from this paper were presented as a poster presentation at the 48th Annual Meeting of the AANS/CNS Section on Pediatric Neurological Surgery in Scottsdale, Arizona, December 5-8, 2019.

\section{Current Affiliations}

Dr. Zamorano-Fernández: Hospital Universitario La Paz, Madrid, Spain.

\section{Correspondence}

Jesse Skoch: Cincinnati Children's Hospital Medical Center, Cincinnati, OH. jesse.skoch@cchmc.org. 